Pacific Journal of Mathematics

ON ESSENTIAL ABSOLUTE CONTINUITY 


\section{ON ESSENTIAL ABSOLUTE CONTINUITY}

\section{ROBERT J. THOMPSON}

Throughout this paper $\boldsymbol{D}$ will denote a bounded domain in Euclidean $n$-space $R^{n}$, and $T$ will be a bounded, continuous, single-valued transformation from $\boldsymbol{D}$ into $R^{n}$. For such transformations, concepts of essential bounded variation and essential absolute continuity have been defined and studied by Rado and Reichelderfer ([3], IV. 4). In this paper a characterization of essential absolute continuity will be given. The characterization suggests a definition of uniform essential absolute continuity and some of the consequences of this definition will be investigated.

1. For every point $x$ in $R^{n}$ a multiplicity function $K(x, T, D)$ is defined ([3], II. 3.2). $T$ is said to be essentially of bounded variation (briefly $e B V)$ in $\boldsymbol{D}$ provided $K(x, T, \boldsymbol{D})$ is Lebesgue summable in $\boldsymbol{R}^{n}$ ([3], IV. 4.1, Definition 1). Let $X_{\infty}=X_{\infty}(T, D)$ denote the set of points $x$ in $R^{n}$ for which $K(x, T, D)$ is infinite. Thus if $T$ is $e B V$ in $D$, then $\mathscr{L} X_{\infty}=0$ (if $A$ is a subset of $R^{n}$, then $\mathscr{L} A$ denotes its exterior Lebesgue measure). Since $K(x, T, D)$ is a lower semicontinuous function of $x$ ([3], II. 3.2, Remark 10), $X_{\infty}$ is a Borel set and, by Theorem 1 of [3], IV. 1.1, the set $T^{-1} X_{\infty}$ is also a Borel set.

2. If $x$ is a point in $R^{n}$ and $C$ is a component of $T^{-1} x$ which is closed relative to $R^{n}$, then $C$ is termed a maximal model continuum (x, $T, D)$ ([3], II. 3.1, Definition 1). Denote by $\mathbb{C}=(5(T, D)$ the class composed of all sets $C$ for which $T C$ is a point in $R^{n}$ and $C$ is a maximal model continuum for $(T C, T, \boldsymbol{D})$. Let $\mathfrak{F}=\mathbb{S}(T, \boldsymbol{D})$ be the subset of $\mathfrak{}$ consisting of those elements $C$ each of which is an essential maximal model continuum (briefly e.m.m.c.) for $(T C, T, D)$ ([3], II. 3.3, Definition 1); the set $E=E(T, D)=\cup C, C \in\left[\right.$ ([3], II. 3.6). Let $\xi_{i}=$

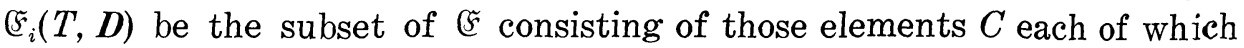
is an essentially isolated e.m.m.c. (briefly e.i. e.m.m.c.) for $(T C, T, D)$ ([3], II. 3.3, Definition 2); the set $E_{i}=E_{i}(T, D)=\cup C, C \in \mathfrak{E}_{i}$ ([3], II. 3.6.). Finally, let $\xi_{i}^{p}=\mathfrak{F}_{i}^{p}(T, D)$ be the subset of $\xi_{i}$ consisting of those elements of $\tilde{F}_{i}$ which consist of single points; the set $E_{i}^{p}=E_{i}^{p}(T, D)=$ $\cup C, C \in \xi_{i}^{p}$ ([3], II. 3.6). The sets $E, E_{i}$ and $E_{i}^{p}$ are Borel sets ([3], II. 3.6, Theorem 1).

If $T$ is $e B V$ in $D$, then a necessary and sufficient condition that $T$ be essentially absolutely continuous (briefly $e A C$ ) in $D$ ([3], IV. 4.2) is

Received December 15, 1960. The results reported here were included in a dissertation presented in partial fulfillment of the requirements for the degree Doctor of Philosophy at The Ohio State University. The author wishes to express his gratitude to Professor P. V. Reichelderfer for his generous help and advice. 
that $T$ satisfies the condition $(N)$ on the set $E(T, \mathrm{D})$ ([3], IV. 4.2, Theorem 3) i.e., if $S \equiv E$ and $\mathscr{L} S=0$, then $\mathscr{L} T S=0$.

DEFINITION 1. $T$ will be said to satisfy the $(\varepsilon, \delta)$ condition on a subset $A$ of $\boldsymbol{D}$ if for every $\varepsilon>0$ there exists a $\delta>0$ such that if $S \equiv A$ and $\mathscr{L} S<\delta$, then $\mathscr{L} T S<\varepsilon$. Clearly if $T$ satisfies the $(\varepsilon, \delta)$ condition on each of a finite number of subsets of $D$, then $T$ satisfies the $(\varepsilon, \delta)$ condition on any subset of their union. Also, if $A$ is a Borel subset of $\boldsymbol{D}$, then $T$ satisfies the $(\varepsilon, \delta)$ condition on $A$ if and only if for every $\varepsilon>0$ there is a $\delta>0$ such that if $S$ is a Borel subset of $A$ and $\mathscr{L} S<\delta$, then $\mathscr{L} T S<\varepsilon$.

Theorem 1. Suppose $T$ is $e B V$ in $D$. Then a necessary and sufficient condition that $T$ be $e A C$ in $\boldsymbol{D}$ is that $T$ satisfies the $(\varepsilon, \delta)$ condition on the set $E(T, D)$.

Proof. Since $T$ is assumed to be $e B V$ in $\boldsymbol{D}$ it suffices to prove that a necessary and sufficient condition that $T$ satisfies the condition $(N)$ on the set $E$ is that $T$ satisfies the $(\varepsilon, \delta)$ condition on $E$. Since the proof of the sufficiency is immediate, we proceed to a proof of the necessity. If $T$ satisfies the condition $(N)$ on $E$, then, by Lemma 4 of [3], IV. 4.2, $\mathscr{L} T\left(E-E_{\imath}^{p}\right)=0$ and so $T$ clearly satisfies the $(\varepsilon, \delta)$ condition on $E-E_{i}^{p}$. Since $T$ is $e B V$ in $D, \mathscr{L} X_{\infty}=0$ and so $T$ satisfies the $(\varepsilon, \delta)$ condition on $T^{-1} X_{\infty}$. Since $E$ is a subset of the union of the sets $E-E_{\imath}^{p}, T^{-1} X_{\infty}$ and $E_{i}^{p}-T^{-1} X_{\infty}$, in view of the remarks following Definition 1 it remains only to be shown that $T$ satisfies the $(\varepsilon, \delta)$ condition on $E_{i}^{p}-T^{-1} X_{\infty}$ whenever $T$ satisfies the condition $(N)$ on $E$. Assume then that $T$ does not satisfy the $(\varepsilon, \delta)$ condition on $E_{i}^{p}-T^{-1} X_{\infty}$. The proof will be completed by showing that $T$ does not satisfy the condition $(N)$ on $E$. Since $E_{i}^{p}$ and $T^{-1} X_{\infty}$ are Borel sets, their difference is a Borel set. Thus the assumption that $T$ fails to satisfy the $(\varepsilon, \delta)$ condition on $E_{i}^{p}-T^{-1} X_{\infty}$ implies, in view of the remarks following Definition 1 , that there is an $\varepsilon_{0}>0$ such that for every positive integer $k$ there is a Borel set $S_{k} \equiv E_{i}^{p}-T^{-1} X_{\infty}$ such that $\mathscr{L} S_{k}<1 / 2^{k}$ and $\mathscr{P} T S_{k} \geqq \varepsilon_{0}$. Let $S^{*}=\lim \sup S_{k}\left(=\bigcap_{n=1}^{\infty} \bigcup_{k \geqq n} S_{k}\right) . \quad S^{*}$ is a subset of $E_{i}^{p}-T^{-1} X_{\infty}$ and so

$$
S^{*} \overline{\bar{C}} E \text {. }
$$

For every positive integer $n, S^{*} \equiv \bigcup_{k \bar{n}} S_{k}$ and so $\mathscr{C} S^{*} ₹ 1 / 2^{n-1}$. Hence

$$
\mathscr{L} S^{*}=0 .
$$

Let $k$ be a positive integer and suppose $x \in T S_{k}$. Since $S_{k} \overline{\bar{C}} E_{i}^{p}-$ $T^{-1} X_{\infty}, K(x, T, D)<\infty$ and there is a point $u$ in $E_{i}^{p}$ such that $T u=x$. 
Since $K(x, T, D)<\infty$ there are at most a finite number of e.m.m.c.s. for $(x, T, D)$ ([3], II. 3.3, Definition 1 and II. 3.4, Theorem 3). But for every point $u$ in $E_{i}^{p}$ such that $T u=x$ the set consisting of the point $u$ is an e.m.m.c. for $(x, T, D)$. Thus there are at most a finite number of points $u$ in $E_{i}^{p}-T^{-1} X_{\infty}$ for which $T u=x$. Thus it has been shown that

(3) For every integer $k$, if $x$ is in $T S_{k}$ then $\left(E_{i}^{p}-T^{-1} X_{\infty}\right) \cap T^{-1} x$ is a finite set.

Since $U S_{k} \bar{\equiv} E_{i}^{p}-T^{-1} X_{\infty}$ it is easy to show that (3) implies that lim sup $T S_{k}=T\left(\lim \sup S_{k}\right)$ and so

$$
\mathscr{L}\left(\lim \sup T S_{k}\right)=\mathscr{L} T S^{*} .
$$

By Theorem 4 of [3], IV. 1. 1, the sets $T S_{k}$ are measurable. Since $T$ is a bounded transformation, $\mathscr{L}\left(\cup T S_{k}\right)$ is finite. Thus ([5], p. 17) $\mathscr{L}\left(\lim \sup T S_{k}\right) \geqq \lim \sup \mathscr{L} T S_{k}$.

But $\mathscr{L} T S_{k} \geqq \varepsilon_{0}>0$ for all $k$ and so

$$
\lim \sup \mathscr{L} T S_{k}>0
$$

By (4), (5) and (6),

$$
\mathscr{L} T S^{*}>0
$$

Now (1), (2) and (7) imply that $T$ does not satisfy condition $(N)$ on $E$.

3. Definition 2. For every positive integer $j$ let $\boldsymbol{D}_{\boldsymbol{j}}$ be a bounded domain in $R^{n}$ and let $T_{j}$ be a bounded, continuous, single-valued transformation from $\boldsymbol{D}_{j}$ into $R^{n}$. The transformations $T_{j}$ will be termed uniformly essentially absolutely continuous (briefly UEAC) provided:

(i) For each $j, T_{j} e B V$ in $\boldsymbol{D}_{j}$ and

(ii) Given any $\varepsilon>0$, there is a $\delta>0$, depending only on $\varepsilon$, such that for all $j$ the following is true: if $S$ is a subset of $E\left(T_{j}, D_{j}\right)$ and $\mathscr{L} S<\delta$, then $\mathscr{L} T_{j} S<\varepsilon$.

Note that if the transformations $T_{j}$ are UEAC, then, by Theorem 1 , for each $j, T_{j}$ is $e A C$ in $\boldsymbol{D}_{j}$.

Each point $u$ in $\boldsymbol{D}$ is contained in a unique component of $T^{-1} T u$ denoted by $C_{u}$. A subset $U$ of $\boldsymbol{D}$ is termed a $T$ set if $u \in U$ implies $C_{u} \equiv U([4], 1)$.

Theorem 2. Let $\boldsymbol{D}$ be a bounded domain in Euclidean $n$-space $R^{n}$ and let $T$ be a bounded, continuous, single-valued transformation from $\boldsymbol{D}$ into $R^{n}$. For every positive integer $j$ let $\boldsymbol{D}_{j}$ be a bounded domain in $R^{n}$ and let $T_{i}$ be a bounded, continuous, single-valued transformation from $\boldsymbol{D}_{j}$ into $R^{n}$, 
If

(i) The mappings $T_{j}$ are UEAC

(ii) The mappings $T_{j}$ converge to $T$ uniformly on compact subsets of $\boldsymbol{D}$ ([3], II. 3. 2, Remark 9) and

(iii) $A$ is a $T$ set contained in $E(T, D)$ and $\mathscr{L} A=0$, then $\mathscr{L} T A=0$.

Proof Let $\varepsilon>0$ be given and let $\delta$ be the corresponding positive number in (ii) of Definition 2. Since $A$ is a subset of the open set $\boldsymbol{D}$ and $\mathscr{L} A=0$, there is an open set $O$, containing $A$ and contained in $D$, such that $\mathscr{L} O<\delta$. Let $x \in T A$. Since $A \equiv E(T, D)$, there is a set $C$, e.m.m.c. for $(x, T, D)$, such that $C$ meets $A$. $C \equiv A$ since $A$ is a $T$ set and so $C \equiv O$. By Definition 1 in [3], II. 3.3 there is a set $D$, which contains $C$ and whose closure $\mathscr{K} D$ is contained in $O$, such that $D$ is an indicator domain for $(x, T, D)$ ([3], II. 3.2). By definition $\mathscr{K} D \equiv D, x$ is not in $T \mathscr{B} D$ (where $\mathscr{B} D$ denotes the boundary of $D$ ) and the topological index $\mu(x, T, D)$ ([3], II. 2) is not zero. Since $T \mathscr{B} D$ is compact, the ecart of $x$ from $T \mathscr{B} D, e(x, T \mathscr{B} D$ ), is positive ([3], I.1.4, Exercise 3). Since $\mathscr{K} D \equiv D$, by (ii) there is a positive integer $j_{x}$ such that, for $j>j_{x}, \mathscr{K} D \equiv D_{j}$ and $\rho\left(T, T_{j}, \mathscr{K} D\right)$ the deviation of $T_{j}$ from $T$ on $\mathscr{K} D$ ([3], I. 1.5, Definition 5) is less than $e(x, T \mathscr{B} D)$. Clearly $\rho\left(T, T_{j}, \mathscr{B} D\right) \leqq \rho\left(T, T_{j}, \mathscr{K} D\right)$. Thus, for $j>j_{x}, \mathscr{K} D \equiv \boldsymbol{D} \cap \boldsymbol{D}_{j}$ and $\rho\left(T, T_{j}, \mathscr{B} D\right)<e(x, T \mathscr{B} D)$. By Theorem 6 of [3], II. 2.3, $\mu\left(x, T_{j}, D\right)$ is defined and equals $\mu(x, T, D)$. Thus $D$ is an indicator domain for $\left(x, T_{j}, D_{j}\right)$ and, by Lemma 4 of [3], II. 3.3, there is a set $C_{j}$, e.m.m.c. for $\left(x, T_{j}, D_{j}\right)$, such that $C_{j} \equiv D$. Now $C_{j} \equiv O \cap E\left(T_{j}, D_{j}\right)$ and $T_{j} C_{j}=x$. Thus $x \in T_{j}\left[O \cap E\left(T_{j}, \boldsymbol{D}_{j}\right)\right]$ for all $j>j_{x}$ and hence $x \in \lim \inf T_{j}[O \cap$ $\left.E\left(T_{j}, D_{j}\right)\right]$. Since $x$ was any point in $T A$, it has been shown that $T A$ $\equiv \lim \inf T_{j}\left[O \cap E\left(T_{j}, \boldsymbol{D}_{j}\right)\right]$ and so

$$
\mathscr{L} T A \leqq \mathscr{L} \lim \inf T_{j}\left[O \cap E\left(T_{j}, \boldsymbol{D}_{j}\right)\right]
$$

Since $E\left(T_{j}, \boldsymbol{D}_{j}\right)$ is a Borel set, $O \cap E\left(T_{j}, \boldsymbol{D}_{j}\right)$ is also a Borel set and so $T_{j}\left[O \cap E\left(T_{j}, \boldsymbol{D}_{j}\right)\right]$ is Lebesgue measurable. Thus ([5], p. 17) $\mathscr{L} \lim \inf T_{j}\left[O \cap E\left(T_{j}, \boldsymbol{D}_{j}\right)\right] \leqq \lim \inf \mathscr{L} T_{j}\left[O \cap E\left(T_{j}, \boldsymbol{D}_{j}\right)\right]$.

Now

$$
\mathscr{L}\left[O \cap E\left(T_{j}, D_{j}\right)\right] \leqq \mathscr{L} O<\delta .
$$

By the choice of $\delta$, (3) implies that $\mathscr{L} T_{j}\left[O \cap E\left(T_{j}, D_{j}\right)\right]<\varepsilon$ and hence $\lim \inf \mathscr{L} T_{j}\left[O \cap E\left(T_{j}, \boldsymbol{D}_{j}\right)\right] \leqq \varepsilon$.

By (1), (2) and (4) 


$$
\mathscr{L} T A \leqq \varepsilon .
$$

Since (5) has been proved for an arbitrary $\varepsilon>0$, it follows that $\mathscr{L} T A=0$.

4. Theorem 2 suggests the question: under the hypotheses of Theorem 2 does $T$ satisfy the condition $(N)$ on $E(T, D)$ ? Note that $T$ does satisfy the condition $(N)$ on $E_{i}^{p}(T, D)$. In the remainder of the paper some results pertinent to this question will be presented.

Reichelderfer introduced the concept of the $T$ magnification ([4], 6). It will be useful to have the definition repeated here.

Let $\mathfrak{D}^{*}=\mathfrak{D}^{*}(T, \boldsymbol{D})$ be the class composed of all domains $D$ for each of which $\mathscr{K} D$ is contained in $\boldsymbol{D}$ and there exists an open oriented $n$-cube $Q$ in $R^{n}$ such that $D$ is a component of $T^{-1} Q$. If $C$ is a maximal model continuum for $(x, T, D)$ for some point $x$ in $R^{n}$, for every positive number $\varepsilon$ define

$$
\bar{d}(C, \mathscr{L} T, \varepsilon)=\text { l.u.b. } \mathscr{L} T D / \mathscr{L} D, C \equiv D \in \mathscr{D}^{*}, \delta T D \leqq \varepsilon
$$

and

$$
\underline{d}(C, \mathscr{L} T, \varepsilon)=\text { g.l.b. } \mathscr{L} T D \mid \mathscr{L} D, C \equiv D \in \mathscr{D}^{*}, \delta T D \leqq \varepsilon
$$

(If $A$ is a subset of $R^{n}, \delta A$ denotes the diameter of $A$ ).

$$
\bar{d}(C, \mathscr{L} T)=\lim _{\varepsilon \rightarrow 0+} \bar{d}(C, \mathscr{L} T, \varepsilon)
$$

and

$$
\underline{d}(C, \mathscr{L} T)=\lim _{\varepsilon \rightarrow 0+} \underline{d}(C, \mathscr{L} T, \varepsilon) .
$$

If $\bar{d}(C, \mathscr{L} T)$ and $\underline{d}(C, \mathscr{L} T)$ are finite and equal, their common value is denoted by $M(C, T)$ and is termed the $T$ magnification at $C$.

Lemma 1. Let $p$ be a positive number and let $A$ be a $T$ set with the following properties:

(i) If $u \in A$, then there is a set $C \in \mathfrak{F}_{i}(T, D)$ such that $u \in C$ and $\underline{d}(C, \mathscr{L} T)>p$.

(ii) If $C \in \mathfrak{F}_{i}(T, D)$ and $C \equiv A$, then for every domain $G$ in $R^{n}$ which contains TC and has a sufficiently small diameter it is true that $T^{-1} G$ possesses exactly one component $D$ which meets $A$. Note that $D$ must contain $C$ and (provided only that the diameter of $G$ is sufficiently small) be a m.i.d. $T$ ([4], 4 and 5, Lemma 2).

Then $\mathscr{L} A \leqq 1 / p \mathscr{L} T A$.

Proof. Let $\eta$ be any positive number. The proof will be completed 
by showing that $\mathscr{L} A \leqq 1 / p \mathscr{L} T A+\eta$.

Let $x \in T A$ (the inequality is trivial if $A$ is empty) and let $u \in A$ such the $T u=x$. By (i) there is a set $C \in \mathfrak{S}_{i}(T, D)$ such that $u \in C$ and $\underline{d}(C, \mathscr{L} T)>p$. Thus there is an $\varepsilon>0$ such that $\underline{d}(C, \mathscr{L} T, \varepsilon)>p$ and so

$$
\text { If } C \equiv D \in \mathfrak{D}^{*} \text { and } \delta T D \leqq \varepsilon \text {, then } \mathscr{L} T D / \mathscr{L} D>p
$$

Since $A$ is a $T$ set, $C \equiv A$ and, by (ii), there exists a positive number $r$ such that for every domain $G$ in $R^{n}$ which contains $T C(=x)$ and for which $\delta G \leqq r$ it is true that $T^{-1} G$ possesses exactly one component which meets $A$ and, moreover, this component is a m.i.d. $T$ containing $C$. For every positive integer $i$ let $Q_{i}$ be the open oriented $n$-cube with center at $x$ and diameter equal to the smaller of $\varepsilon, r$ and $1 / i$. Then $T^{-1} Q_{i}$ possesses exactly one component $D_{i}$ which meets $A$ and $D_{i}$ is a m.i.d. $T$ containing $C$. By the Lemma in [4], 4, $T D_{i}=Q_{i}$ and $\mathscr{K} D_{i} \equiv D$. By definition, $D_{i} \in \mathfrak{D}^{*}$ and so, with the aid of (1), $\mathscr{L} D_{i}<1 / p \mathscr{L} T D_{i}$. Thus

(2) For every point $x$ in $T A$ there is associated a sequence of open oriented $n$-cubes $Q_{i}$ with centers at $x$ and a corresponding sequence of domains $D_{i}$ such that, for all $i, \delta Q_{i} \leqq 1 / i, \mathscr{L} D_{i},<1 / p \mathscr{L} Q_{i}, D_{i}$ is a component of $T^{-1} Q_{i}$ and the only component of $T^{-1} Q_{i}$ which meets $A$.

Let $\cong$ be the class of all $n$-cubes associated with points of $T A$ in this manner. $\mathscr{L} T A$ is finite since $T$ is bounded, and by a theorem of

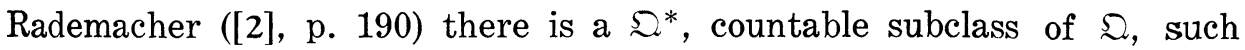
that

$$
T A \equiv \cup Q^{*}, Q^{*} \in \mathfrak{\Omega}^{*}
$$

and

$$
\Sigma \mathscr{L} Q^{*} \leqq \mathscr{L} T A+\eta p .
$$

(Rademacher's theorem is stated in terms of a covering made up of open $n$-spheres, but the corresponding theorem for a covering of open $n$-cubes is readily obtained from it). Let $Q^{*}$ be an element of $\Omega^{*}$. By (2) there is a corresponding domain $D^{*}, D^{*}$ a component $T^{-1} Q^{*}$ such that $\mathscr{L} D^{*}<1 / p \mathscr{L} Q^{*}$ and $D^{*}$ is the only component of $T^{-1} Q^{*}$ which meets $A$. In this way exactly one domain $D^{*}$ is associated with each $Q^{*} \in \mathfrak{\Omega}^{*}$. The class of domains $D^{*}$ is countable and

$$
\Sigma \mathscr{L} D^{*} \leqq 1 / p \Sigma \mathscr{L} Q^{*} \text {. }
$$

Let $u \in A$. Then $T u \in T A$ and by (3) there is a $Q^{*} \in \mathfrak{Q}^{*}$ such that $T u \in Q^{*}$. Since the corresponding $D^{*}$ is the only component of $T^{-1} Q^{*}$ 
which meets $A$ it must contain $u$. Thus $A \equiv \cup D^{*}$ and

$$
\mathscr{L} A \leqq \Sigma \mathscr{L} D^{*} \text {. }
$$

By (4), (5) and (6), $\mathscr{L} A \leqq 1 / p \mathscr{L} T A+\eta$. Since $\eta$ is any positive number, the conclusion of the lemma is established.

Lemma 2. Let $\mathfrak{T}$ be a subclass of $\mathfrak{F}_{i}(T, D)$ such that if $C \in \mathfrak{T}$ then $\underline{d}(C, \mathscr{L} T)>0$. Put $H=\cup C, C \in \mathfrak{S}$. If $\mathscr{L} T H=0$, then $\mathscr{L} H=0$.

Proof. If $H$ is not empty (the equality is trivial otherwise) then $\mathfrak{F}_{i}(T, D)$ is not empty and hence, by the Lemma in [4], 14, the set $E_{i}$ can be expressed as the union of a countably infinite sequence of $T$ sets $U_{k}$ with the following property:

(1) If $C \in \dot{\xi}_{i}$ and $U_{k} \equiv C$, then for every domain $G$ in $R^{n}$ which contains $T C$ and has a sufficiently small diameter it is true that $T^{-1} G$ possesses exactly one component $D$ which meets $U_{k}$.

For every positive integer $n$ let $\mathfrak{S}_{n}$ be the subclass of $\mathfrak{S}$ consisting of those elements $C$ for which $\underline{d}(C, \mathscr{L} T)>1 / n$. Put $H_{n}=\cup C, C \in \mathfrak{S}_{n}$ and let $H_{n k}=H_{n} \cap U_{k}$. Then $H=\cup H_{n}$ and, for each $n, H_{n}=\cup H_{n k}$. The proof will be completed by showing that $\mathscr{L} H_{n k}=0$ for arbitrary $n$ and $k$. Since $H_{n}$ and $U_{k}$ are $T$ sets,

(2) $H_{n k}$ is a $T$ set.

Clearly

(3) If $u \in H_{n k}$, then there is a set $C \in \mathfrak{F}_{i}$ such that $u \in C$ and $\underline{d}(C, \mathscr{L} T)>1 / n$.

By (1) and the definition of $H_{n k}$,

(4) If $C \in \xi_{i}$ and $C \equiv H_{n k}$, then for every domain $G$ in $R^{n}$ which contains $T C$ and has a sufficiently small diameter it is true that $T^{-1} G$ possesses exactly one component $D$ which meets $H_{n k}$.

(2), (3), (4) and Lemma 1 imply that $\mathscr{L} H_{n k} \leqq n \mathscr{L} T H_{n k}$. Since $T H_{n k} \equiv T H$ and $\mathscr{L} T H=0, \mathscr{L} T H_{n k}=0$ and consequently $\mathscr{L} H_{n k}=0$. Since $n$ and $k$ are arbitrary, it follows that $\mathscr{L} H=0$.

5. Theorem 3. Let $\boldsymbol{D}$ be a bounded domain in Euclidean n-space $R^{n}$ and let $T$ be a bounded, continuous, single-valued transformation from $\boldsymbol{D}$ into $R^{n}$. For every positive integer $j$ let $\boldsymbol{D}_{j}$ be a bounded domain in $R^{n}$ and let $T_{j}$ be a bounded, countinuous, single-valued transformation from $\boldsymbol{D}_{j}$ into $R^{n}$. Let $\mathfrak{B}$ be the subclass of $\mathfrak{F}_{i}(T, D)$ 
consisting of those elements $C$ for each of which $C M(C, T)$ exists and is positive and $C$ contains more than a single point. Put $B=\cup C$, $C \in \mathfrak{B}$. If

(i) The mappings $T_{j}$ are UEAC.

(ii) The mappings $T_{j}$ converge to $T$ uniformly on compact subsets $D$ and

(iii) $T$ is $e B V$ in $\boldsymbol{D}$

then the following statements are equivalent:

(iv) $T$ satisfies the condition $(N)$ on $B$,

(iv)' $\mathscr{L} T B=0$ and

(iv)" $\mathscr{L} B=0$

and (i), (ii) and (iii) together with (iv) or (iv)' or (iv)" imply that $T$ is $e A C$ in $D$.

Proof. First it will be shown that (i), (ii), (iii) and (iv) imply that $T$ is $e A C$ in $D$. By the Theorem in [4], 16, there exist $T$ sets $V^{\prime}$ and $V^{\prime \prime}$ contained in $\boldsymbol{D}$ such that $\mathscr{L} V^{\prime}=0, \mathscr{L} T V^{\prime \prime}=0$ and if $C \in \mathfrak{F}_{i}(T, \boldsymbol{D})$ and $C$ does not meet $V^{\prime} \cup V^{\prime \prime}$, then $M(C, T)$ exists and is positive. In view of (iii), in order to conclude that $T$ is $e A C$ in $D$ it is sufficient to prove that $T$ satisfies the condition $(N)$ on $E=E(T, D)$. Clearly it is sufficient to show that $T$ satisfies the condition $(N)$ on each of the following sets whose union is $E: S_{1}=E-E_{i}, S_{2}=E_{i}^{p}, S_{3}=\left(E_{i}-\right.$ $\left.E_{i}^{p}\right) \cap V^{\prime}, S_{4}=\left(E_{i}-E_{i}^{p}\right) \cap V^{\prime \prime}$ and $S_{5}=\left(E_{i}-E_{i}^{p}\right)-\left(V^{\prime} \cup V^{\prime \prime}\right)$. Since $T$ is $e B V$ in $D, \mathscr{L} T S_{1}=0$ (this is proved in the first step in the proof of the theorem in [4], 18) and so $T$ satisfies the condition $(N)$ on $S_{1}$. Any subset of $S_{2}$ is a $T$ set contained in $E$ and it follows by Theorem 2 that $T$ satisfies the condition $(N)$ on $S_{2}$. Again by Theorem 2, $\mathscr{L} T S_{3}=0$ and so $T$ satisfies the condition $(N)$ on $S_{3}$. $\mathscr{L} T S_{4} \leqq \mathscr{L} T V^{\prime \prime}=0$ and so $T$ satisfies the condition $(N)$ on $S_{4} . S_{5}$ is a subset of $B$ and so (iv) implies that $T$ satisfies condition $(N)$ on $S_{5}$.

If (i), (ii), (iii) and (iv) are satisfied, then it has just been shown that $T$ satisfies the condition $(N)$ on $E(T, D)$. Hence, by Lemma 4 of [3], IV. $4.2, \mathscr{L} T\left(E-E_{i}^{p}\right)=0$. Since $B$ is a subset of $E-E_{i}^{p}$, (iv)' must be satisfied. On the other hand, (iv)' clearly implies (iv). Thus if (i), (ii) and (iii) are satisfied, (iv) and (iv)' are equivalent.

By Lemma $2, \mathscr{L} B=0$ if $\mathscr{L} T B=0$. On the other hand, since $B$ is a $T$ set contained in $E(T, D)$, (i) and (ii) imply, by Theorem 2, that $\mathscr{L} T B=0$ if $\mathscr{L} B=0$. Hence if (i) and (ii) are satisfied, then (iv)' and (iv)" are equivalent.

6. It is reasonable to inquire whether (i), (ii) and (iii) in Theorem 3 are sufficient to conclude that $T$ is $e A C$ in $D$. After all, each of the sets $C$ in $\mathfrak{B}$ is a non-point continuum for which the $T$ magnification is 
positive and yet whose image under $T$ is a single point in $R^{n}$. Might not (i), (ii) and (iii) imply, say, (iv)' (or equivalently (iv) or (iv)")? Since the class $\mathscr{B}$ is clearly countable when $T$ is a transformation into $R^{1}, T B$ is then a countable set. Thus (iv)' is always satisfied when $T$ is a transformation into $R^{1}$. However, the author has constructed an example in $R^{2}$ for which (i), (ii) and (iii) are satisfied and for which the limit transformation is not $e A C([6])$. In the example the limit transformation $T$ is modeled on an example by Cesari ([1], IV. 13.1, Example A). The transformation that Cesari defined provides an example of a plane mapping that is $e B V$ but not $e A C$. The example in [6] is somewhat more complicated by the need for (i) and (ii) to be satisfied.

\section{BIBLIOGRAPHY}

1. L. Cesari, Surface Area, Annals of Mathematics Studies, No. 35, Princeton University Press, 1956.

2. H. Rademacher, Eineindeutige Abbildungen und Messbarkeit, Monatshefte fur Mathematik und Physik, 27 (1916), 183-290.

3. T. Rado, and P. Reichelderfer, Continuous Transformations in Analysis, Die Grundlehren der Mathematischen Wissenschaften, Vol. 75, Springer-Verlag, 1955.

4. P. Reichelderfer, A Study of the Essential Jacobian in Transformation Theory, Rendiconti del Circolo Matematico di Palermo, Series 2, 6 (1957), 175-197.

5. S. Saks, Theory of the Integral, Monografie Matematyczne, Warsaw, 1937.

6. R. Thompson, On Essential Absolute Continuity for a Transformation, Dissertation, The Ohio State University, 1958 (L. C. Card No. Mic 58-3467).

Sandia Corporation, Albuquerque, New Mexico 



\section{PACIFIC JOURNAL OF MATHEMATICS}

\section{EDITORS}

\author{
RaLPh S. Phillips \\ Stanford University \\ Stanford, California \\ F. H. BRowNELL \\ University of Washington \\ Seattle 5 , Washington
}

A. L. Whiteman

University of Southern California

Los Angeles 7, California

L. J. Paige

University of California

Los Angeles 24, California

\author{
E. F. BECKENBACH \\ T. M. CHERRY
}

\author{
ASSOCIATE EDITORS

$\begin{array}{lll}\text { D. DERRY } & \text { H. L. ROYDEN } & \text { E. G. STRAUS } \\ \text { M. OHTSUKA } & \text { E. SPANIER } & \text { F. WOLF }\end{array}$

\section{SUPPORTING INSTITUTIONS}

\author{
UNIVERSITY OF BRITISH COLUMBIA \\ CALIFORNIA INSTITUTE OF TECHNOLOGY \\ UNIVERSITY OF CALIFORNIA \\ MONTANA STATE UNIVERSITY \\ UNIVERSITY OF NEVADA \\ NEW MEXICO STATE UNIVERSITY \\ OREGON STATE COLLEGE \\ UNIVERSITY OF OREGON \\ OSAKA UNIVERSITY \\ UNIVERSITY OF SOUTHERN CALIFORNIA
}

\author{
STANFORD UNIVERSITY \\ UNIVERSITY OF TOKYO \\ UNIVERSITY OF UTAH \\ WASHINGTON STATE COLLEGE \\ UNIVERSITY OF WASHINGTON \\ AMERICAN MATHEMATICAL SOCIETY \\ CALIFORNIA RESEARCH CORPORATION \\ HUGHES AIRCRAFT COMPANY \\ SPACE TECHNOLOGY LABORATORIES \\ NAVAL ORDNANCE TEST STATION
}

Mathematical papers intended for publication in the Pacific Journal of Mathematics should be typewritten (double spaced), and the author should keep a complete copy. Manuscripts may be sent to any one of the four editors. All other communications to the editors should be addressed to the managing editor, L. J. Paige at the University of California, Los Angeles 24, California.

50 reprints per author of each article are furnished free of charge; additional copies may be obtained at cost in multiples of 50 .

The Pacific Journal of Mathematics is published quarterly, in March, June, September, and December. The price per volume (4 numbers) is $\$ 12.00$; single issues, $\$ 3.50$. Back numbers are available. Special price to individual faculty members of supporting institutions and to individual members of the American Mathematical Society: $\$ 4.00$ per volume; single issues, $\$ 1.25$.

Subscriptions, orders for back numbers, and changes of address should be sent to Pacific Journal of Mathematics, 103 Highland Boulevard, Berkeley 8, California.

Printed at Kokusai Bunken Insatsusha (International Academic Printing Co., Ltd.), No. 6, 2-chome, Fujimi-cho, Chiyoda-ku, Tokyo, Japan.

\section{PUBLISHED BY PACIFIC JOURNAL OF MATHEMATICS, A NON-PROFIT CORPORATION}

The Supporting Institutions listed above contribute to the cost of publication of this Journal, but they are not owners or publishers and have no responsibility for its content or policies.

Reprinted 1966 in the United States of America 


\section{Pacific Journal of Mathematics}

\section{Vol. 11, No. 4}

A. V. Balakrishnan, Prediction theory for Markoff processes . . . . . . . . . . 1171

Dallas O. Banks, Upper bounds for the eigenvalues of some vibrating systems . . . . 1183

A. Białynicki-Birula, On the field of rational functions of algebraic groups ...... 1205

Thomas Andrew Brown, Simple paths on convex polyhedra .............. 1211

L. Carlitz, Some congruences for the Bell polynomials . . . . . . . . . . . . 1215

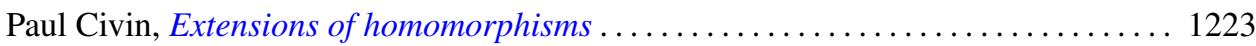

Paul Joseph Cohen and Milton Lees, Asymptotic decay of solutions of differential

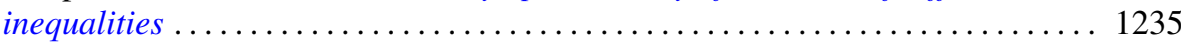

István Fáry, Self-intersection of a sphere on a complex quadric . . . . . . . . . . 1251

Walter Feit and John Griggs Thompson, Groups which have a faithful representation

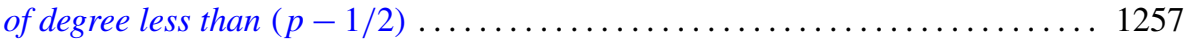

William James Firey, Mean cross-section measures of harmonic means of convex

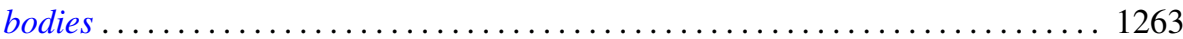

Avner Friedman, The wave equation for differential forms . . . . . . . . . . 1267

Bernard Russel Gelbaum and Jesus Gil De Lamadrid, Bases of tensor products of

Banach spaces ................................... 1281

Ronald Kay Getoor, Infinitely divisible probabilities on the hyperbolic plane . . . . 1287

Basil Gordon, Sequences in groups with distinct partial products . . . . . . . . . . . . 1309

Magnus R. Hestenes, Relative self-adjoint operators in Hilbert space . . . . . . . . . 1315

Fu Cheng Hsiang, On a theorem of Fejér ......................... 1359

John McCormick Irwin and Elbert A. Walker, On N-high subgroups of Abelian

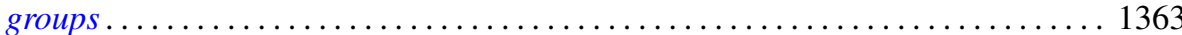

John McCormick Irwin, High subgroups of Abelian torsion groups . . . . . . . . . 1375

R. E. Johnson, Quotient rings of rings with zero singular ideal . . . . . . . . . . . 1385

David G. Kendall and John Leonard Mott, The asymptotic distribution of the time-to-escape for comets strongly bound to the solar system ...

Kurt Kreith, The spectrum of singular self-adjoint elliptic operators ....

Lionello Lombardi, The semicontinuity of the most general integral of the calculus of variations in non-parametric form ................................

Albert W. Marshall and Ingram Olkin, Game theoretic proof that Chebyshev inequalities are sharp

Wallace Smith Martindale, III, Primitive algebras with involution . . William H. Mills, Decomposition of holomorphs ..............

James Donald Monk, On the representation theory for cylindric algebras . . . . . . 1447

Shu-Teh Chen Moy, A note on generalizations of Shannon-McMillan theorem . . . . 1459

Donald Earl Myers, An imbedding space for Schwartz distributions . .

John R. Myhill, Category methods in recursion theory .........

Paul Adrian Nickel, On extremal properties for annular radial and circular slit mappings of bordered Riemann surfaces

Edward Scott O'Keefe, Primal clusters of two-element algebras . .

Nelson Onuchic, Applications of the topological method of Wazewski to certain

problems of asymptotic behavior in ordinary differential equations ...

Peter Perkins, A theorem on regular matrices................

Clinton M. Petty, Centroid surfaces .... 